FORMATION Formation emploi

Revue française de sciences sociales

97 | janvier-mars 2007

Former pour dynamiser les territoires

\title{
Les intermédiaires de proximité, pour inciter les pme à former
}

Local mediators inciting SMEs to dispense vocational training

Lokale Förderung der betrieblichen Berufsbildung

\section{Marc Lecoutre}

\section{(2) OpenEdition}

Journals

Édition électronique

URL : http://journals.openedition.org/formationemploi/1664

DOI : 10.4000/formationemploi. 1664

ISSN : 2107-0946

Éditeur

La Documentation française

Édition imprimée

Date de publication : 1 janvier 2007

Pagination : 23-35

ISSN : 0759-6340

Référence électronique

Marc Lecoutre, "Les intermédiaires de proximité, pour inciter les pme à former », Formation emploi [En ligne], 97 | janvier-mars 2007, mis en ligne le 31 mars 2009, consulté le 30 octobre 2020. URL : http:// journals.openedition.org/formationemploi/1664; DOI : https://doi.org/10.4000/formationemploi.1664

(c) Tous droits réservés 


\title{
DOSSIER
}

\section{Les intermédiaires de proximité, pour inciter les PME à former}

Par Marc Lecoutre*

\author{
La volonté de stimuler, au niveau local, des opérateurs légitimes et compétents \\ en matière de formation professionnelle, reconnus et disponibles \\ dans l'environnement des entreprises, apparaît justifiée. Cela soulève \\ néanmoins plusieurs interrogations.
}

La décentralisation de la formation professionnelle, engagée depuis près de trente ans (Bel, Méhaut, Mériaux, 2003), et son mouvement corollaire de déconcentration impliquent aujourd'hui des acteurs de plus en plus nombreux. Ceci s'inscrit dans une évolution plus générale des modes d'intervention de l'État, au plus près des réalités des cibles de ses politiques (Simonin, 1995 ; Lallement, 1996), et dans la perspective d'un «pilotage rapproché» (Méhaut, 2003). L'État peut ainsi mobiliser des acteurs de proximité au niveau territorial dans la mise en œuvre de ses politiques de formation professionnelle, distincts de ses propres services déconcentrés (Brochier, Lecoutre, 2000). On désigne ainsi un ensemble d'acteurs présents sur les territoires, le plus souvent organisés autour d'une branche professionnelle ${ }^{1}$. La pièce maîtresse de ce système est la branche professionnelle, secondée sur le terrain, dans le cas de la formation professionnelle, par son organisme paritaire collecteur agréé (OPCA). Ce dernier assure des prestations de services directement à ses adhérents, et

${ }^{1}$ regroupement d'employeurs s'organisant collectivement autour d'une profession ou d'un métier donné. prend en charge des relations croissantes avec nombre d'instances techniques ou politiques à caractère public, en passant par l'ensemble des institutions ou partenaires du marché de la formation.

* Marc Lecoutre est sociologue, enseignant-chercheur en gestion des ressources humaines au Groupe ESC Clermont (centre régional associé au Céreq) et LISE (Laboratoire interdisciplinaire de sociologie économique). Ses travaux portent sur le rôle des réseaux sociaux dans le pilotage de l'action organisée, et le capital social des organisations éducatives. II a récemment publié : "Le capital social dans les transitions écoles-entreprises » in Bevort, Lallement (Éds.), Le capital social-Performance, équité et réciprocité, la Découverte, coll. "Bibliothèque du Mauss », 2006 ; avec Anne Moisan-Louazel et Gérard Podevin, "Les relations des services de proximité avec les territoires et les petites entreprises » in Bentabet E., Théry M. (Éds), Les OPCA, acteurs du changement des comportements des petites entreprises en matière de formation professionnelle, Céreq-Relief, n 1 1, 2006. 
L'idée d'intermédiaire ou d'intermédiation au niveau local peut être mobilisée pour désigner cette façon d'agir (Simonin, 1995; Bessy, Eymard-Duvernay, 1997). Certes, résultat d'une histoire très centralisée $\mathrm{du}$ système français de relations professionnelles (Lallement, 1996), les branches professionnelles restent peu enclines à s'organiser régionalement et localement (Giraud, Mériaux, 2003 ; Culpepper, 2003) ; cependant, elles demeurent incontournables étant donné leur poids financier et organisationnel dans le champ de la formation professionnelle (Géhin, 2003). Ainsi, la mobilisation des branches professionnelles a pris du sens dans la pratique de l'État (Brochier, Lecoutre, 2000). En parallèle, l'idée qu'il fallait se rapprocher des entreprises, et singulièrement des PME, s'est peu à peu développée. Quels sont les enjeux de ce type de fonction au niveau local, que ce soit auprès des entreprises ou de façon plus générale envers l'État? Nous l'analysons ici à partir de l'exemple d'un dispositif du ministère du Travail, les engagements de développement de la formation $(\mathrm{EDDF})^{2}$, complété par des éléments d'analyse issus d'une étude sur l'implication locale des OPCA (encadré 1). Dans un premier temps, nous rappelons quels sont l'objectif et le fonctionnement du dispositif des EDDF et nous précisons en quoi consiste cette fonction d'intermédiation locale dans le champ de la formation, et ses caractéristiques. Nous identifions ensuite quelques enjeux liés au rôle actif joué par l'intermédiaire au niveau local. Nous appliquons enfin dans les parties 3 et 4 cette réflexion au cas des trois branches étudiées.

\section{MOBILISER DES INTERMÉDIAIRES PROCHES DES ENTREPRISES}

L'objectif du dispositif des EDDF est de stimuler le développement de la formation continue au sein des petites et moyennes entreprises (PME) à l'aide de subventions incitatives au plan de formation (Verdier, Brochier, 1997). Nous en rappelons ici le principe puis sa spécificité au plan territorial.

\footnotetext{
${ }^{2}$ Depuis janvier 2006, le dispositif des EDDF a été remplacé par celui des EDEC (engagements de développement de l'emploi et des compétences). Il fusionne en particulier le dispositif d'étude préalable à la conclusion d'un EDDF (CEP, contrats d'études prospectives) avec celui des EDDF (décret $\mathrm{n}^{\circ}$ 2006-54 du 16 janvier 2006 paru au JO du 18/01/06).
}

\section{Encadré 1}

Sources et méthode

Cette contribution revisite une étude réalisée en 1996-1997 sur la politique contractuelle de formation professionnelle (Brochier, Lecoutre 2000 ; Verdier, Brochier, 1997), en s'appuyant sur les questionnements d'un travail sur la proximité territoriale OPCA PME (Organisme paritaire collecteur agréé petites et moyennes entreprises) et en montrant les différentes formes d'implication de ces OPCA au niveau territorial (Lecoutre, Moisan-Louazel, Podevin, 2006 ; Bentabet, Théry, Éds, 2006). La première étude avait porté sur trois branches: la métallurgie, anciennement structurée et extrêmement organisée; la plasturgie, branche jeune et en pleine structuration à l'époque; et la grande distribution alimentaire, présente au niveau national mais très faiblement organisée au niveau local, malgré la grande répartition territoriale de ses unités. La seconde étude s'interrogeait pour sa part sur la capacité des OPCA à initier des changements significatifs dans les comportements des PME en matière de formation professionnelle, et a interrogé plus particulièrement les cas de trois OPCA de branche (Bâtiment, FAF Propreté et Promofaf - devenu entretemps Uniformation -) et d'un OPCA interprofessionnel, I'AGEFOS-PME (*). Les deux études se sont appuyées sur une analyse documentaire historique et un ensemble d'entretiens approfondis avec les divers acteurs des dispositifs et organismes étudiés. L'auteur remercie par ailleurs F. Dauty, M. Bel et les membres du groupe Céreq-centres associés "Formation et territoire », ainsi que D. Brochier pour leurs relectures et remarques.

$\left(^{*}\right)$ : Organisme paritaire collecteur agréé, non spécifique à un secteur d'activité donné, chargé du financement et du conseil en matière de formation professionnelle pour les PME et leurs salariés. 


\section{Un dispositif pour développer} la formation continue

Cette forme d'intervention de l'État a vu le jour au début de la décennie 80 , et a constitué le premier instrument de la politique contractuelle de l'État en matière de formation continue ${ }^{3}$. Il consiste en une aide financière de l'État au développement de la formation dans une entreprise, soumise à négociation et à passation d'un contrat entre les deux parties, imposant le respect d'un certain nombre de critères. L'entreprise doit réaliser une programmation pluriannuelle des actions de formation. Son engagement doit correspondre à une augmentation nette de ses dépenses de formation par rapport aux années antérieures. Enfin, le plan de formation envisagé doit être à la fois ambitieux en matière d'élévation des compétences, et cohérent avec la stratégie d'évolution technologique, économique ou organisationnelle de l'entreprise. L'État, en contrepartie, s'engage à contribuer financièrement selon des taux de prise en charge négociés. Enfin, les institutions représentatives du personnel de l'entreprise sont consultées afin de donner leur avis sur le projet de formation aidé ${ }^{4}$.

Centré sur la construction de liens directs entre les entreprises et l'État, ce dispositif a transformé ses modes d'intervention en s'appuyant, à partir de 1989, sur les branches professionnelles ${ }^{5}$. Alors que jusqu'à présent les grandes entreprises avaient constitué l'essentiel des cibles, l'État souhaite atteindre plus massivement les petites et moyennes entreprises (PME). Il prend alors conscience de la difficulté de la tâche. Malgré une certaine proximité de ses services déconcentrés au niveau territorial, les PME apparaissent relativement éloignées de l'environnement culturel étatique sur ce champ particulier de la formation continue en entreprise et de son articulation à leur développement (Bentabet, Michun, Trouvé, 1999 ; Bentabet, Michun, 2003).

\footnotetext{
${ }^{3}$ Selon le préambule de la circulaire fondatrice de 1984, les EDDF « sont destinés aux entreprises assujetties à l'obligation légale de participation au développement de la formation professionnelle qui accroissent, en quantité et en qualité, leur effort de formation dans le cadre d'un programme pluriannuel dont les objectifs sont partagés par l'État et les partenaires sociaux 》.

${ }^{4}$ Gérard et alii, 1993 ; Verdier, 1990 ; circulaire DFP n 96/11 du 16 juillet 1996.

${ }^{5}$ Mirochnitchenko et Verdier, 1997 ; circulaires DFP n ${ }^{\circ} 902 \mathrm{du}$ 3 mars $1989, \mathrm{n}^{\circ} 1767$ du 5 juin 1989, de 1993, de 1995, DFP n 96/11 du 16 juillet 1996.
}

L'État décide alors de mobiliser des acteurs-relais spécifiques, perçus comme plus efficaces pour entrer en contact avec les cibles visées. Ce seront les organismes professionnels du secteur ou de la branche spécialisés dans la formation et proches des entreprises au niveau territorial. Ces organismes sont chargés par convention de repérer les entreprises susceptibles de prétendre à une aide de l'État pour développer leur programme de formation continue, de les aider à monter le dossier d'aide et à présenter celui-ci auprès de l'instance d'attribution. Ce n'est donc plus un représentant de l'État qui agit directement sur le terrain pour inciter les entreprises à développer leur formation avec des aides financières, mais une structure professionnelle de branche, désignée au niveau national, au sein d'accords de branche déclinés ensuite au niveau régional pour l'action publique déconcentrée.

Si l'architecture administrative des EDDF est à plusieurs niveaux (Brochier, Lecoutre, 2000), l'essentiel du travail se réalise au niveau territorial dans l'environnement des entreprises-cibles. Cellesci sont mobilisées dans la phase de réalisation d'un dossier correspondant au maximum aux priorités nationales. Elles sont sélectionnées par les techniciens des organismes (des conseillers-formation par exemple) qui, au sein de chaque branche, ont été désignés pour cela. Ainsi, il peut s'agir des chambres syndicales territoriales de la métallurgie pour les entreprises relevant de l'UIMM (Union des industries métallurgiques et minières), mais les OPCA sont le plus souvent impliqués: Associations régionales pour la formation dans le BTP (AREF), structures territoriales de l'AGEFOS-PME6 pour l'accord interprofessionnel, par exemple. Ce travail de « recrutement» des entreprises est essentiel : c'est sur ces techniciens que repose la tâche d'identification des entreprises potentiellement porteuses de projets de formation ou simplement réceptives au développement de celle-ci. Mais ils se trouvent aussi en situation de devoir opérer une certaine traduction des textes et orientations définies dans les circulaires nationales et les accords régionaux, de construire une sorte d'équivalence conventionnelle entre des pratiques

\footnotetext{
${ }^{6}$ Organisme paritaire collecteur agréé, non spécifique à un secteur d'activité donné, chargé du financement et du conseil en matière de formation professionnelle pour les PME et leurs salariés.
} 


\section{Encadré 2}

\section{Les engagements de développement de la formation, une organisation multiniveaux}

Un accord EDDF (engagement de développement de la formation) est conclu entre l'État et une organisation professionnelle, un regroupement d'entreprises ou une entreprise. II encadre les aides publiques versées aux entreprises qui s'engagent, sur plusieurs années, à accroître, en qualité et en quantité, leur effort de formation.

Son fonctionnement est le suivant :

- une convention-cadre entre l'État et une organisation professionnelle de branche au niveau national formalise des objectifs et fixe des moyens pour le développement des efforts de formation des entreprises appartenant à la branche ;

- chaque accord-cadre national peut être décliné dans des conventions régionales d'application conclues entre la Direction régionale du Travail, de l'Emploi et de la Formation professionnelle (DRETFP, représentant l'État) et les services de l'organisation professionnelle de branche à ce niveau, avec aménagement éventuel par rapport aux spécificités régionales ;

- l'instance de débat et de décision est le Comité technique régional d'attribution des aides (CTRA), présidé par l'État, dont le travail est organisé par les DRTEFP, et auquel d'autres institutions peuvent être associées selon les cas;

- les conseils régionaux peuvent intervenir en cofinancement de l'EDDF dès lors que le projet ou une partie de celui-ci correspond à des objectifs de développement qu'ils ont définis comme prioritaires. De même, l'aide de l'État peut être complétée par des cofinancements du Fond social européen (FSE).

Le travail sur le terrain auprès des entreprises est mené ainsi :

- l'entité régionale ou locale possédant la compétence technique en matière de formation (I'OPCA Organisme paritaire collecteur agréé, - le plus souvent) se voit déléguer le travail de repérage des entreprises et d'aide à la construction du dossier de demande d'aide ; en tant qu'organisme conseil de l'entreprise, elle identifie les PME (petites et moyennes entreprises) éligibles dans le cadre de son activité de soutien permanent à propos de leur plan de formation ;

- les Directions départementales du Travail, de l'Emploi et de la Formation professionnelle réceptionnent, vérifient la conformité et instruisent les dossiers de demande d'aide présentés par ces entreprises jusqu'à la présentation de ceux-ci dans les instances régionales.

d'entreprises, par essence multiformes et relevant de logiques privées, et les attentes des acteurs publics locaux en charge de l'attribution des aides.

\section{Les nécessaires médiations territoriales}

Pour rendre compte de la fonction assurée par ces organismes et leurs techniciens dans le champ de la formation professionnelle, la notion d'intermédiaire (Vincens, 1995) apparaît plus éclairante que celle d'acteurs relais, supposant une certaine neutralité qui s'avère illusoire. Cette notion apparaît de plus en plus souvent dans le champ de l'emploi ou de la formation, et recouvre certes des situations assez différentes. Elle semble enfin faire l'objet d'une reconnaissance comme thème de recherche émergent et d'un consensus sur la nécessité d'en approfondir l'analyse dans le champ de l'action publique « [...] parce que la caractérisation de ces dynamiques intermédiaires est loin d'être acquise $»^{7}$. Nous retenons ici comme définition les phénomènes de mobilisation par l'État d'acteurs situés hors de sa propre sphère, ancrés territorialement et intervenant dans la mise en œuvre de ses politiques de formation professionnelle.

On peut ainsi identifier trois caractéristiques de cette fonction d'intermédiation en matière de formation.

\footnotetext{
${ }^{7}$ In Les dynamiques intermédiaires. Approches interdisciplinaires, texte de présentation du colloque organisé à Toulouse les 16 et 17 septembre 2004 par le CERTOP.
} 
D'abord, ce n'est jamais un phénomène spontané ou ponctuel, dans la mesure où l'acteur ou l'organisme qui l'assure a été délibérément choisi par l'État pour être intermédiaire de sa politique. Il s'inscrit dans le cadre des relations structurelles qui s'établissent entre des organisations porteuses d'intérêts sociaux et l'État (Mény, Thoenig, 1989). Ensuite, il ne s'agit pas d'une simple relation de sous-traitance, puisque le service proposé par l'intermédiaire est autant construit par l'État (et ses différents services) que par l'intermédiaire lui-même. L'autonomie - par essence - de l'acteur intermédiaire dans ses relations négociées avec l'État l'amène à intervenir dans la construction de la politique. Et enfin, cet acteur est supposé offrir une structuration au niveau territorial et une inscription locale qui le rendent apte à atteindre ses cibles dans la mesure où il doit suppléer à une relative absence de l'État.

Dans l'exemple des EDDF présenté ici, le ministère du Travail a décidé de confier aux branches professionnelles le soin d'atteindre les PME relevant de leur champ. Ces intermédiaires, organisés au niveau national, sont perçus comme ayant développé une proximité territoriale avec les PME et comme étant spécialisés - entre autres - dans le champ du dispositif public, la formation professionnelle en entreprise. À ce titre, ils apparaissent capables d'entrer aisément en contact avec elles pour leur fournir le service adéquat (Verdier 1990 ; Lecoutre, MoisanLouazel, Podevin, 2006). Cette capacité de proximité avec les cibles de l'EDDF suppose alors une présence organisée au niveau territorial et local, répondant en cela à la personnalisation des liens et l'inscription dans des réseaux locaux caractéristiques du fonctionnement de nombre de PME (Bentabet, Michun, 2003 ; Michun, 2007). Ainsi, ces acteurs de la branche professionnelle apparaissent devoir être dépositaires d'une double légitimité : acteurs intermédiaires spécialisés mobilisés par et pour une politique publique de formation professionnelle initiée par l'État d'un coté ; mais aussi de l'autre, ressource effective et reconnue de l'environnement proche des cibles, les PME, et mobilisée par celles-ci dans leur fonctionnement habituel. Après le rappel de leurs spécificités, il s'agit de s'interroger sur les enjeux de ces pratiques d'intermédiation.

\section{LES ENJEUX DES PRATIQUES D'INTERMÉDIATION LOCALE POUR L'ÉTAT}

L'étude de 1996-1997 s'appuyait sur une analyse de trois branches professionnelles: la métallurgie, la plasturgie et la grande distribution alimentaire. Elle a montré que l'exercice d'une fonction d'intermédiation par une branche professionnelle en matière de politique de formation professionnelle n'était pas neutre quant à la régulation de l'action publique (Brochier, Lecoutre, 2000). Nous reprenons ici certains des éléments évoqués pour montrer comment cette fonction d'intermédiation, conçue de manière homogène par l'État, est confrontée à deux enjeux qui déterminent l'un et l'autre l'effectivité de sa fonction d'intermédiaire de proximité : d'un coté, le pouvoir d'infléchissement de l'intermédiaire local quand il est très structuré et, de l'autre, la faiblesse de sa consistance territoriale.

\section{Intermédiaires et jeux de pouvoir}

Par essence, au plan formel, l'intermédiaire est dans une position qui lui confère un pouvoir potentiel conséquent. L'analyse des réseaux sociaux a bien montré comment un tiers pouvait tirer avantage de sa posture d'intermédiaire dans une triade (Degenne et Forsé, 1994). Dans le cas traité ici, la branche professionnelle développe une stratégie d'articulateur (lorsque l'intermédiaire connecte deux mondes sociaux) plutôt que de tertius gaudens («troisième larron ») tirant un pouvoir de la compétition entre ses deux contacts : elle met en effet en relation deux intermédiés n'ayant pas (ou peu) de relations entre eux (l'État et les PME), à l'initiative de l'un d'entre eux (l'État), plutôt qu'elle ne cherche à tirer parti de conflits existant entre les deux intermédiés. Cette stratégie d'articulateur est bien celle que l'État cherche à lui confier, cohérente en cela avec le rôle de représentant ${ }^{8}$ que la branche professionnelle occupe vis-à-vis des entreprises. Mais si ce rôle de représentant est l'une des positions possibles pour

\footnotetext{
${ }^{8}$ Reprenant les travaux de Gould et Fernandez, Degenne et Forsé présentent cinq rôles possibles pour un articulateur: l'intermédiaire, le représentant, le gardien, le médiateur et le coordinateur, selon l'appartenance ou non de l'articulateur et des individus qu'il relie aux mêmes cercles sociaux (1994, p. 150).
} 
l'intermédiaire, il peut, de façon tout à fait symétrique, se considérer comme un gardien de l'accès à ceux qu'il représente (Degenne, Forsé, 1994, pp. 149151). L'État est donc l'initiateur de la fonction d'intermédiation dévolue aux branches au niveau territorial. Comment peut-il alors contrôler et encadrer l'activité de ces acteurs intermédiaires dans la réalisation de cette fonction? Quel type de pouvoir leur est confié ?

L'analyse, à travers les textes des circulaires, des compétences accordées par l'État à l'intermédiaire permet de mesurer le degré d'autonomie dont ce dernier dispose dans la conduite du dispositif. L'État cherche, dans sa relation avec l'intermédiaire, à garder le contrôle de la relation de celui-ci avec les cibles du dispositif, et à cadrer très strictement sa nature. Il conserve certes la maitrise des flux financiers qui constituent le moteur du dispositif (Mirochnitchenko, Verdier, 1997). Mais il s'est surtout doté de deux leviers principaux : il est d'une part l'énonciateur des critères prioritaires de sélection des entreprises, des conditions d'attribution des aides. Ces critères font bien entendu l'objet de précisions et de formulations spécifiques négociées au sein de chaque accord de branche, ils sont discutés et adaptés aux spécificités locales dans les conventions régionales d'applications, mais ils doivent s'inscrire dans le canevas global tracé par la circulaire et respecter les orientations considérées comme prioritaires (en particulier la formulation des termes de l'engagement d'une entreprise demandeuse). D'autre part, l'État offre à ses agents les moyens d'infléchir à chaque instant le cours du déroulement de sa politique, en particulier l'identification et l'accompagnement des cibles au niveau local. La formule selon laquelle «les représentants de l'État peuvent, dans les comités d'attribution des aides, imposer l'examen de la demande présentée par une entreprise entrant dans le champ d'application d'un accord et disposent d'un droit de veto au versement de l'aide à une entreprise $»^{9}$ est à cet égard explicite.

L'autonomie potentielle de l'acteur intermédiaire se manifeste cependant clairement dans la seconde relation de la fonction d'intermédiation locale, celle le liant aux cibles du dispositif public et donnant tout

${ }^{9}$ circulaire DFP n ${ }^{\circ}$ 96/11 du 16 juillet 1996, p 10. son sens à son caractère de proximité. La question qui se pose alors est celle du maintien du caractère public de la politique. Les risques d'absorption dans le cadre plus général de la stratégie de l'acteur intermédiaire ne peuvent être ignorés. Les marges de manœuvre apparaissent parfois suffisamment larges pour permettre l'élaboration d'une stratégie propre, en partie distincte de celle des pouvoirs publics (encadré 3). Dans le cas de la politique contractuelle de formation, les objectifs des EDDF sont censés être congruents avec ceux de la branche, mais risquent de se trouver englobés dans les enjeux plus larges de la relation entre une organisation de branche et les entreprises qu'elle représente. De son association avec une organisation professionnelle, l'État attend en effet beaucoup. Sans que cela soit explicitement formulé, ces organisations de branches sont ainsi perçues comme de véritables experts sectoriels du service visé par l'État: «[les] conventions de branche conclues au niveau national [...] permettent d'appréhender globalement les problèmes de formation qui se posent face à une stratégie de développement et d'adaptation d'un milieu professionnel et de toucher un grand nombre d'entreprises qui appartiennent à ce milieu $»^{10}$. Cela laisse cependant de coté le fait que les organismes composant ces branches, et implantés au niveau local, sont aussi offreurs d'autres services aux entreprises visées ${ }^{11}$ et que ceux-ci ne sont pas forcément tous gratuits. Cette situation peut alors contribuer à stimuler outre mesure la recherche d'entreprises répondant aux critères de sélection, au détriment de la qualité des opérations aidées, favorisant du même coup les effets d'aubaine.

\section{Définition et structuration territoriale des acteurs}

Sur la base de quelle organisation interne, de quelle structuration territoriale une branche professionnelle est-elle en mesure de remplir les missions d'intermédiation locale qui lui sont confiées par l'État?

\footnotetext{
${ }^{10}$ circulaire n 1767 du 5 juin 1989, article III-1.

${ }^{11}$ Comme par exemple les conseils juridiques divers sur l'évolution de la législation en matière salariale, sociale, fiscale, etc., l'information sur la mise en place de formations adaptées à leurs besoins, la définition des besoins de formation dans l'entreprise, ou encore l'aide à la construction du plan de formation. Ces tâches sont en général assurées par des conseillers salariés des chambres syndicales, parfois appelés conseillers-formation lorsqu'ils sont spécialisés sur ce champ.
} 


\section{Encadré 3}

\section{Entre logique " marchande » et logique de " service public », le difficile positionnement des acteurs de terrain}

À titre d'exemple, le travail réalisé auprès des OPCA (organismes paritaires collecteurs agréés) a permis de classer leurs interventions par ordre croissant d'importance : fourniture d'informations administratives, techniques, financières, juridiques (remplissage des déclarations 24.83, construction des cotisations, mécanismes de prise en charge...) ; conseil technique au montage du plan de formation, aide au choix des prestataires; enfin, conseil en gestion des compétences s'apparentant nettement à du conseil d'entreprise opéré par des consultants (Lecoutre, Moisan-Louazel, Podevin, 2006). Voici ce que nous avons pu observer (Bentabet, Théry, (Éds.), 2006, p. 39) :

"Certains OPCA, éloignés de toute pratique commerciale, et dont les adhérents relèvent de fait d'un marché captif compte tenu de l'obligation conventionnelle de versement, souhaitent clairement situer ces prestations nouvelles ou en développement dans une logique dite de "service public ", à charge pour eux de trouver les financements. Mais la contrainte financière limite le nombre possible de bénéficiaires, et/ou nécessite des ajustements sur d'autres prestations dont le poids devra être réduit.

Pour d'autres, il est admis que de toute façon les services de conseil et d'accompagnement individualisés ne peuvent fonctionner pour l'instant que parce qu'un nombre limité d'entreprises en font la demande. Toutefois, la recherche de nouveaux adhérents (souvent assimilés à des "clients») devient une stratégie qui, par augmentation des sommes collectées et donc aussi des frais de gestion, doit faciliter le financement de ces services. La recherche de subventions, notamment européennes, pour des activités de conseil éligibles au regard des critères du FSE ou de fonds régionaux mobilisables pour des opérations collectives mais qui permettent une réorientation de certains budgets vers des actions personnalisées, est souvent complémentaire au développement des portefeuilles d'adhérents. Ici la référence à la proximité renvoie d'une part à des pratiques effectives de nouveaux services rendus et d'autre part à une logique de marketing visant à conquérir de nouvelles parts de marché. Dans ce cas, la concurrence entre OPCA est réelle, que ce soit entre OPCA interprofessionnels, ou entre OPCA interprofessionnels et OPCA de branche. »

Dans le système français de relations professionnelles, la branche a toujours été instaurée comme partenaire principal de l'État dans son dialogue avec les entreprises (Saglio, 1991; Lallement, 1996). Le cas des EDDF interroge ici cette conception quasi «mythique » de la branche et révèle ici des problèmes et des ambigüités de deux types : la prise en compte des acteurs syndicaux dans sa définition, et l'organisation effective des organismes de branche au niveau territorial.

Tout d'abord, la branche peut n'être prise en compte que dans son sens classique strict, c'est-à-dire comme le regroupement d'employeurs s'organisant collectivement autour d'une profession ou d'un métier donné. Mais elle peut aussi inclure le partenaire syndical en intégrant le résultat des négociations avec celui-ci, cristallisé notamment dans des accords collectifs. C'est ce second sens qui prédomine pour l'acteur public. Certes, les conventions d'EDDF sont passées exclusivement entre des employeurs (ou des représentants d'employeurs) et l'État, mais ce dernier considère implicitement les organisations syndicales de salariés comme des partenaires de la procédure. Leur consultation dans les différentes instances paritaires de concertation aux niveaux national, régional ou d'entreprise est prévue dans les textes. De même, leur association au sein des instances de suivi du dispositif est suggérée, sans pour autant prendre un caractère obligatoire ${ }^{12}$. Enfin, leur présence au niveau national interprofessionnel est reconnue, à travers la participation de leurs

${ }_{12}$ "Quoique l'article L.951.5 du Code du Travail ne prévoit pas l'association des organisations syndicales de salariés représentatives au suivi des engagements de développement de la formation, il apparait nécessaire de la prévoir si la demande s'exprime en ce sens. » (circulaire DFP n' 96/11 du 16 juillet 1996, p 10). 
représentants au sein de l'instance nationale de pilotage de la politique. L'intervention des syndicats de salariés dans le dispositif est ainsi soutenue par une conception d'un système idéal de relations professionnelles dans lequel les partenaires sociaux seraient à même d'établir un dialogue constructif, à tous les échelons géographiques (national, régional, local), autour de la gestion d'un objet commun, justifiant ainsi la mobilisation des OPCA dans ce dispositif.

Le second point concerne l'ancrage territorial de la branche, c'est-à-dire l'existence d'un degré de structuration susceptible de rendre effective son action comme intermédiaire de proximité. La branche est en effet créditée, dans le champ de la formation professionnelle, d'une véritable capacité de mobilisation des PME qui composent son secteur professionnel, indépendamment de la réalité de sa structuration territoriale. C'est la question incontournable du cadre territorial dans lequel un intermédiaire doit, selon l'État, inscrire son action. Le postulat est qu'en provoquant un rapprochement des représentants locaux de l'État et de l'acteur intermédiaire, on améliore le pilotage de la politique publique. Présent de façon implicite dans la seconde moitié de la décennie 90 , ce thème de la proximité est devenu depuis un mot d'ordre quasi obligé de toute action publique qui se veut crédible ${ }^{13}$. Les textes supposent une organisation de la branche particulièrement adaptée au suivi du dispositif. Ils postulent l'existence d'un ensemble de pôles régionaux ayant un ancrage local, à la fois étroitement connectés à l'organisation nationale qui doit rester le pôle d'impulsion, et surtout dotés d'une présence effective sur le territoire et d'une certaine autonomie afin de contextualiser au mieux le dispositif ${ }^{14}$. L'État imagine finalement une structuration des branches de type national/régional/ local, quelles que soient les spécificités de chacune d'elles, ceci révélant de sa part une vision assez normative de ce que doit être l'intermédiaire puisqu'il cherche en quelque sorte à le contraindre jusque dans son mode d'organisation au niveau territorial.

${ }^{13} C f$. les $3^{e}$ et $4^{\mathrm{e}}$ éditions des Journées de la Proximité organisées par le groupe de recherche "Dynamiques de proximité " et en 2001 (Paris) avec l'université de Paris XI (ADIS), l'INRA (SAD); en 2004 (Marseille) avec le Laboratoire d'économie et de sociologie du travail (LEST), le Groupement de recherche en économie quantitative (Greqam) et l'Institut d'économie publique (IDEP).

${ }^{14}$ Ce qui ne semble pas forcément aller de soi, comme l'a montré l'étude sur les OPCA (Bentabet, Théry 2006).

\section{TROIS EXEMPLES D'INTERMÉDIATION SELON LA STRUCTURATION DE LA BRANCHE}

Les pratiques observées dans trois branches et deux régions (Rhône-Alpes et Provence-Alpes-Côte d'Azur) engagées dans la politique contractuelle de formation, révèlent des configurations différenciées de l'activité d'intermédiation locale de la part des branches. La métallurgie est historiquement une branche très puissante, réunissant 1,8 million de salariés pour 20000 entreprises adhérentes. Sa base territoriale est extrêmement structurée et compte 93 chambres syndicales territoriales (CST) réunissant les employeurs du secteur, dont la taille varie d'un grand bassin d'emploi, lorsqu'il y a une forte concentration d'entreprises de ce secteur, à un ou deux départements. Elle a ainsi développé une intermédiation de type « captatif» (Brochier, Lecoutre, 2000), s'inscrivant dans une proximité effective avec ses adhérents. Elle n'a jamais intégré la logique des EDDF, s'appropriant pendant un temps la gestion des fonds, pesant de tout son poids en maintenant le cap d'une vision de la formation comme variable stratégique relevant uniquement des employeurs et non des salariés, d'où la mobilisation de ses unités locales de la branche, les CST. Celles-ci, très proches de leurs adhérents au niveau local, jouent auprès des entreprises un rôle de conseiller dans de nombreux domaines, en particulier celui de la formation. Ainsi, à la différence d'autres branches, ces CST avaient déjà, avant la mise en œuvre des EDDF, à la fois une structuration locale puissante et une activité très développée, même si les EDDF ont été l'occasion d'élargir leurs activités donc leur légitimité - sur un champ, celui de la formation, encore assez neuf. Pour les conseillersformation spécialisés de ces CST, l'EDDF a représenté un atout parmi d'autres dans la palette des services offerts aux entreprises. Ces dernières ont donc en général connu le dispositif à travers leur action décisive, avec le risque de noyer l'idée d'aide publique au milieu d'interventions relevant uniquement de la branche. Le fait qu'il s'agisse d'une aide de l'État a ainsi pu échapper à l'entreprise, l'intermédiaire pouvant à l'occasion faire « écran » (cf. le rôle de «gardien» évoqué plus haut). Le pouvoir de l'intermédiaire apparaît ici déterminant. De même, la 
métallurgie n'offre pas de structuration régionale véritable, d'un niveau décisionnaire équivalent à celui de l'échelon régional du ministère du Travail (Direction régionale du Travail, de l'Emploi et de la Formation professionnelle): les CST ont seules le pouvoir, certaines jouant le jeu d'une véritable coordination régionale quand d'autres ont pu s'ériger en seules destinataires des aides attribuées au niveau régiona ${ }^{15}$.

La Plasturgie est une branche jeune, dont l'unification de la fédération patronale ne remonte qu'à la fin des années 80. Ceci explique son souci constant de légitimation institutionnelle, aussi bien auprès des pouvoirs publics que des entreprises (Besucco et alii, 1998). Cette branche avait ainsi tout intérêt, dans sa recherche de légitimité, à se conformer au rôle attribué par l'État: elle est confrontée à la nécessité d'élever nettement le niveau de qualification de sa main-d'œuvre ${ }^{16}$ afin d'accompagner son fort mouvement d'investissement et de faire face à une forte croissance, et ce rôle d'intermédiaire de proximité lui permet aussi de gagner en légitimité auprès des entreprises. L'intermédiation a donc tout à fait correspondu aux attentes de l'État. La prise en compte de la formation dans le dialogue social, à travers le dispositif des EDDF, a constitué pour la branche une réelle opportunité et correspondait à sa volonté de renforcer son identité professionnelle (cf. le rôle de « représentant » évoqué plus haut) et de développer son organisation territoriale (Saglio, 1991). La Plasturgie, aussi bien du point de vue des fédérations patronales que des organisations syndicales, s'est en fait structurée en partie autour de ce rôle d'acteur intermédiaire des politiques publiques de formation, trouvant dans le dispositif un levier pour son propre développement. La mobilisation par la branche de l'organisme collecteur paritaire (OPCA Plastifaf) et son implication très marquée territorialement, auprès des PME d'un secteur composé en grande partie d'ouvriers non qualifiés et ayant de gros besoins de formations, a été déterminante au plan de la constitution d'une expertise rapidement acquise et reconnue par tous. Pour la Fédération de la

\footnotetext{
${ }^{15}$ Comme dans le cas de la région PACA où la CST d'un seul département était engagée à l'époque dans les dispositifs (Brochier Lecoutre, 2000)

$1670 \%$ d'ouvriers non qualifiés lors de la signature du premier accord cadre en 1985 .
}

Plasturgie, l'EDDF a été un moyen intéressant pour s'organiser localement et consolider son identité en tant qu'organisation fédérative d'employeurs en utilisant la formation comme une ressource dont les entreprises ont particulièrement besoin. Pour les syndicats, l'enjeu plus prosaïque a été celui de l'implantation dans un secteur d'activité faiblement syndiqué ${ }^{17}$, car composé en majeure partie de PME et dépourvu d'une tradition revendicative ouvrière.

Enfin, à l'opposé des deux branches précédentes, l'intermédiation locale s'est avérée limitée dans la branche de la Grande Distribution alimentaire. Bien que les instances nationales de cette dernière aient manifesté une adhésion forte au principe des EDDF, la faible structuration de la branche au niveau territorial ne lui a pas permis de se positionner comme intermédiaire de proximité, limitant le nombre d'entreprises touchées. Cette branche se caractérise en effet par une adhésion forte à la dynamique d'intervention publique en jouant un rôle actif pour la mise en œuvre des EDDF dans les entreprises du secteur entre 1992 et 1995. Cependant, l'observation détaillée de l'impact de l'accord-cadre montre que celui-ci n'a pas réussi clairement à dépasser un « noyau dur » d'entreprises, très impliquées dans les instances de la principale organisation professionnelle du secteur (Verdier, Brochier, 1997). Ce noyau correspond aux grandes entreprises très présentes dans les instances de pilotage de la branche au niveau national, et aux quelques entreprises de taille plus réduites situées dans leur environnement proche. $\mathrm{Au}$ delà de ce cercle restreint, le rôle d'intermédiaire de la branche semble avoir été beaucoup plus difficile à tenir. L'organisation interne du secteur a joué en sa défaveur. En effet, en dépit d'efforts réalisés à la demande des pouvoirs publics pour régionaliser l'accord, en créant des instances de décision dans trois régions, la régionalisation $\mathrm{du}$ dispositif débouche sur un échec complet ${ }^{18}$ et amène à concentrer l'ensemble de la gestion des dossiers sur le seul niveau national. L'explication de cette situation est à rechercher d'une part dans l'organisation interne d'un secteur d'activité dominé par quelques grands

\footnotetext{
${ }^{17}$ La CGT (Confédération générale du travail) ne compterait par exemple que 150 sections syndicales dans le secteur, pour environ 4400 établissements.

${ }^{18}$ Dans une seule des trois régions, un comité régional d'attribution des aides s'est réuni, une seule fois. (Verdier, Brochier, 1997).
} 
groupes intégrés, aux unités implantées partout sur le territoire, mais aux centres de décision extrêmement concentrés au niveau national; d'autre part et, de façon corollaire, dans l'absence de structures de l'organisation professionnelle au niveau territorial.

\section{PROXIMITÉ TERRITORIALE ET RELATION DE POUVOIR}

S'il convient d'éviter toute généralisation hâtive à partir d'une analyse nécessairement partielle et localisée, on retiendra à propos de cette fonction d'intermédiation locale l'instabilité du compromis entre efficacité accrue des politiques publiques et comportements opportunistes des branches, et surtout le poids des dimensions structurelles territoriales dans l'effectivité de la fonction. Ainsi, on peut considérer que le dialogue et les compromis noués au niveau national entre l'État et les acteurs centraux des branches lors de la mise en œuvre de la politique contractuelle de formation professionnelle ont largement contribué à rapprocher les points de vue des protagonistes. La branche a ainsi été positionnée comme une instance de médiation ayant en charge une part de la régulation du marché du travail à travers la promotion de stratégies de formation adaptées (Besucco, Tallard, Lozier, 1998).

Cependant, deux phénomènes intriqués ont finalement joué sur la diffusion effective d'une fonction de médiation censée stimuler les pratiques de formation professionnelle dans les entreprises au niveau local: d'un coté, l'hétérogénéité de la structuration des branches professionnelles interroge leur légitimité vis-àvis de cette fonction; de l'autre, les organisations de branche mobilisées au niveau local autour de cet objectif ont été confrontées à la question de l'articulation entre la logique d'institution privée dont elles sont porteuses et la logique d'action publique qui leur est confiée. Si l'intermédiaire doit partager une même définition de la situation avec ses intermédiés pour assurer correctement sa mission, il est clair que, sur le thème de la formation, la proximité des organismes mobilisés sur le terrain - les OPCA de plus en plus souvent - avec les entreprises fait pencher la balance de leur coté. Mais cette proximité est en même temps un «mal nécessaire » puisqu'elle est la source de la légitimité et de la compétence recherchées par l'État dans sa mobilisation d'un intermédiaire.

Ainsi, l'organisation patronale de la métallurgie a pu correspondre, pour une bonne part, aux attentes des pouvoirs publics en matière de proximité territoriale, même si le niveau régional est relativement absent. Dans le même temps, les conseillers-formation des CST sont des opérateurs tout à fait pertinents pour une politique qui souhaite atteindre les PME faiblement outillées du point de vue de la formation et plutôt éloignées de l'administration. Cependant, le fait de devoir rendre des comptes à leur commanditaire public, en particulier sur le nombre d'entreprises bénéficiaires, tout en étant sensible à l'augmentation du nombre des adhérents, a pu parfois générer des comportements potentiellement opportunistes dans la sélection des cibles, et donc concourir à ce que l'importance du nombre d'entreprises atteint par le dispositif devienne un enjeu au détriment de la pertinence des choix opérés et de la qualité des opérations aidées. De plus, selon Culpepper (2003, p. 82), la branche de la Métallurgie est exemplaire des associations professionnelles trop uniquement orientées sur une relation bilatérale de type « service à l'adhérent », tout comme celle du Bâtiment d'ailleurs (Lecoutre, Moisan-Louazel, Podevin 2006). Malgré - ou à cause ? - d'une structuration locale forte, ces organisations restent plutôt rétives au développement d'une capacité de délibération qui appréhenderait les besoins des entreprises en les mutualisant pour les faire valoir auprès des acteurs publics de niveau régional au poids grandissant en matière de formation professionnelle (conseils régionaux, DRTEFP).

La grande distribution alimentaire s'est certes caractérisée par une grande qualité de dialogue au niveau national. Mais celle-ci a contrasté avec l'absence nette de représentation territoriale des intérêts de la branche. Le constat est celui de la difficulté du dispositif d'aide public à initier un mode d'organisation de la branche plus territorialisé pour toucher véritablement d'autres entreprises que celles que ce dispositif mobilisait antérieurement. Certes, le passage d'une logique de branche «nationale verticale - économique » à une logique de territoire «spatialisée - horizontale - sociale» semble se dessiner (Géhin 2003, p. 32), mais il se heurte autant 
à l'inertie du système de relations professionnelles qu'aux formes de structuration des entreprises composant cette branche.

Enfin, c'est dans la Plasturgie que, semble-t-il, les compromis les plus viables ont pu être trouvés entre les attentes des pouvoirs publics et les objectifs des entreprises du secteur, à partir d'un objet commun, la formation professionnelle, qui devient alors la source d'un mouvement d'organisation territoriale de l'intermédiaire. Le dispositif des EDDF a ainsi obtenu un résultat indirect ${ }^{19}$ et probablement irréversible, la stimulation de l'organisation de la branche au niveau territorial et son gain en proximité, en même temps que la reconnaissance de la professionnalité de son OPCA. Cette branche représente le partenaire idéal apprécié des pouvoirs publics en ayant développé une capacité à être présente au double niveau national et territorial, et en s'appuyant sur un engagement conjoint et constructif d'un noyau bien identifié de partenaires patronaux et syndicaux.

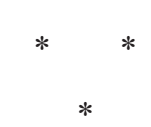

Nous terminerons ici en nous interrogeant brièvement sur la question de la proximité. On reconnaitra que le besoin de se rapprocher des cibles de ses politiques semble devenir incontournable pour l'État aujourd'hui. Cette question de la proximité est en fait un mouvement générique qui concerne l'ensemble de la société (Lallement, 1998). Ainsi, les transformations des systèmes éducatif et productif, depuis le milieu de la décennie 70 , ont généré une société plus fluide, plus décentralisée, plus en réseau et moins hiérarchisée. Les nouvelles formes de régulation se construisent davantage sur l'autonomie et le face-à-face, les stratégies et négociations locales. L'État et les grandes institutions de niveau national occupent toujours une place centrale. Mais dans le même temps, ils confient tous à des acteurs de proximité et dans de nombreux champs (école, justice, police, urbain, services administratifs... et bien sûr formation) le soin de s'ajuster entre eux, de piloter les changements qui les concernent et de produire les normes propres à leurs contextes locaux. Ce retour vers une proximité locale et la confiance qui lui est associée est parallèlement renforcé par l'importance croissante d'un environnement international toujours plus prégnant pour les entreprises, et singulièrement les PME, dépassant les cadres territoriaux traditionnels (Boisard, Courault, 2000 ; Courault, Trouvé, 2001).

La volonté de susciter la présence, au niveau local, d'opérateurs légitimes et compétents en matière de formation professionnelle, reconnus et disponibles dans l'environnement des entreprises, apparaît donc justifiée de ce point de vue, s'inscrivant dans la tendance des évolutions actuelles. Et la revendication par les acteurs collectifs de certaines branches d'une plus grande proximité avec les entreprises, au-delà d'une volonté de re-légitimer leur action, est plus qu'un simple slogan. Mais la " proximité » est-elle en soi toujours une bonne chose? Risquons une critique. La proximité envahit les discours de façon consensuelle et elle serait par évidence toujours vertueuse. On peut replacer cette notion dans un contexte plus large de perception d'un relâchement généralisé du lien social, comme le souligne Lefebvre (2004). Nombre d'institutions collectives d'encadrement chercheraient à légitimer à nouveau leur action en mettant en avant l'idée de proximité, selon l'équation : " proximité = implication = participation $=$ efficacité = légitimité ». Pour cet auteur, "Le fétichisme de la proximité et l'idée que la vérité sociale git dans la "base" et le "terrain" traversent de nombreuses régions de l'espace social. La proximité est considérée comme une valeur sociale refuge dans un monde pensé comme sans repères, impersonnel, anomique, complexe... Réservoir de solutions, elle est unanimement célébrée pour ses vertus pacificatrices, réparatrices, unifiantes, impliquantes» (Lefebvre, 2004, p. 167). Il ne s'agissait pas ici de condamner cet engouement aussi bien des organismes des branches professionnelles que de l'État et de ses services pour inciter au développement d'acteurs de proximité dans le champ de la formation professionnelle, mais bien plutôt de rappeler en quoi cet engouement s'inscrit finalement dans un mouvement plus large, auquel il participe, mais qui le dépasse et imprègne la société tout entière.

${ }^{19}$ Ou peut-être de « second rang » ? 
Bel M., Méhaut P., Mériaux O. (coord.) (2003), La décentralisation de la formation professionnelle. Quels changements dans la conduite de l'action publique?, L'Harmattan, coll. « Logiques Politiques », 252 p.

Bentabet E., Michun S. (2003), «Petites entreprises et réseaux : quelle intermédiation pour la formation continue?», Formation Emploi, n ${ }^{\circ}$ 84, Paris, La Documentation française.

Bentabet E., Michun S., Trouvé Ph. (1999), "Gestion des hommes et formation dans les très petites entreprises », Marseille, Céreq, coll. «Études », n 72, janvier, $175 \mathrm{p}$.

Bentabet E., Théry M. (Éds) (2006), Les OPCA, acteurs du changement des comportements des petites entreprises en matière de formation professionnelle, Marseille, Céreq-Relief, $\mathrm{n}^{\circ} 11$.

Bessy C., Eymard-Duvernay F. (dir.) (1997), «Les intermédiaires du marché du travail», Cahier $d u$ Centre d'Études de l'Emploi, n³6, PUF, 416 p.

Besucco N., Tallard M., Lozier F. (1998), « Politique contractuelle de formation et négociation collective de branche", Travail et Emploi, ministère de l'Emploi et de la Solidarité, La Documentation française, $132 \mathrm{p}$.

Boisard P., Courault B. (2000), « Les dynamiques des PME : du local à l'international », 4 Pages du Centre d'Études de l'Emploi $\mathrm{n}^{\circ}$ 42, décembre.

Brochier D., Lecoutre M. (2000), «La mobilisation des branches professionnelles dans la mise en œuvre des politiques publiques. Le cas des engagements de développement de la formation », La Revue de l'IRES, n' 32.

Courault B., Trouvé Ph. (Dirs) (2001), «Les dynamiques des PME. Approches internationales », PUF, Les Cahiers du Centre d'Études de l'Emploi, $\mathrm{n}^{\circ} 38,488 \mathrm{p}$.
Culpepper P. (2003), "L'information privée et les politiques publiques en perspective comparative $»$, in Bel M., Méhaux P., Mériaux O., La décentralisation de la formation professionnelle, L'Harmattan, coll. « Logiques Politiques », pp. 69-84.

Degenne A., Forsé M. (1994), Les réseaux sociaux, Paris, A. Colin.

Géhin J.-P. (2003), «À la recherche de modalités locales de régulation", in Bel M., Méhaut $\mathrm{P}$., Mériaux O., La décentralisation de la formation professionnelle, L'Harmattan, coll. «Logiques Politiques », pp. 21-41.

Gérard D., Lhotel H., Mouy P., Serfaty E. (1993), Contribution à l'évaluation des engagements de développement de la formation - Rapport final, tome 1, Céreq, Marseille.

Giraud O., Mériaux O. (2003), « France et Allemagne : décentralisation et capacité de régulation des systèmes d'acteurs régionaux », in Bel M., Méhaut P., Mériaux O., La décentralisation de la formation professionnelle, L'Harmattan, coll. « Logiques Politiques », pp. 43-67.

Lallement M. (1996), Les relations professionnelles, Paris, La Découverte, coll. « Repères », 128 p.

Lallement M. (1998), «Les métamorphoses des systèmes éducatifs et productifs", in Galland O., Lemel Y. (Éds), La nouvelle société française. Trente années de mutations, Paris, A. Colin, pp. 117-145.

Lecoutre M., Moisan-Louazel A., Podevin G. (2006), «Relations des services de proximité avec les territoires et les petites entreprises », in Bentabet E., Théry M. (Éds), Les OPCA, acteurs du changement des comportements des petites entreprises en matière de formation professionnelle, Marseille, Céreq-Relief, $\mathrm{n}^{\circ} 11$, pp. 33-48.

Lefebvre R. (2004), La "proximité»: nouveau capital social au chevet de la démocratie?, Actes du 
colloque «Le Capital social», 06/02/03, Cellule Gris, n 10, université de Rouen, pp. 167-199.

Méhaut P. (2003), «Introduction générale 》, in Bel M., Méhaut P., Mériaux O., La décentralisation de la formation professionnelle, L'Harmattan, coll. «Logiques Politiques», pp. 7-15.

Meny Y., Thoenig J.-C. (1989), Politiques publiques, PUF, $391 \mathrm{p}$.

Michun S. (2007), « Petites entreprises et territoire, un lien surestimé ?», contribution à ce numéro 97 de Formation Emploi: revue française de sciences sociales, à paraître.

Mirochnitchenko K., Verdier É. (1997), «Contrat et action publique. Le cas de la formation professionnelle continue », Travail et Emploi n ${ }^{\circ}$ 72, pp. 51-66.
Saglio J. (1991), «La régulation de branche dans le système français de relations professionnelles", Travail et Emploi, n 47, pp. 26-41.

Simonin B. (Dir.) (1995), « Les politiques publiques d'emploi et leurs acteurs ", Cahier du Centre d'Études de l'Emploi, n ${ }^{\circ}$ 34, Paris, PUF.

Verdier É. (1990), «L'efficacité de la formation continue dans les PME », Sociologie du travail, 3/90, pp. 295-320.

Verdier É., Brochier D. (Éds) (1997), Les aides publiques à la formation continue dans les entreprises: quelles modalités d'évaluation?, Céreq, Document $n^{\circ} 124$, série «Évaluation », 254 p.

Vincens J. (1995), « L'intermédiation dans le domaine du travail et de l'emploi ", note 181 (95-2), LIRHE, université des sciences sociales de Toulouse, $12 \mathrm{p}$.

\section{Résumé}

\section{Les intermédiaires de proximité, pour inciter les PME à former \\ Marc Lecoutre}

À travers l'effet structurant des engagements de développement de la formation (EDDF), l'État cherche à faire jover à la branche professionnelle, via son OPCA (Organisme paritaire collecteur agréé), un rôle de proximité accru dans le développement de la formation professionnelle, en particulier en direction des petites et moyennes entreprises (PME). Dans certains cas, I'État n'échappe pas toujours au comportement opportuniste de l'OPCA. Dans d'autres, ce développement de la formation professionnelle pourrait devenir la source d'un mouvement d'organisation territoriale de I'OPCA, ce qui n'est pas toujours confirmé dans les pratiques. Enfin, l'attrait pour la proximité n'est pas indépendant d'une idéologie qui pare cette dernière de toutes les vertus.

Mots clés

Approche locale, formation professionnelle en entreprise, OPCA - Organisme paritaire collecteur agréé, PME-PMI, EDDF.

Journal of Economic Literature: R58, M53, R3 
